\title{
Triple antibiotic therapy with ceftolozane/tazobactam, colistin and rifampin for pan-resistant Pseudomonas aeruginosa ventilator-associated pneumonia
}

\author{
Ammar Alqaid MD, Christopher K. Dougherty DO, Shahbaz Ahmad MD
}

\begin{abstract}
Emergence of multi-drug resistant microorganisms, such as pan-resistant Pseudomonas aeruginosa, has recently created a therapeutic challenge in ICU patients worldwide. New antipseudomonal antibiotics, like ceftolozane/tazobactam, have been developed to meet this challenge. This drug does not demonstrate cross-resistance with other antimicrobial classes, like carbapenems, because of its enhanced binding affinity to the penicillin-binding proteins. A Phase III, multicenter, prospective, randomized, double blind study has been initiated to evaluate the safety and efficacy of ceftolozane/tazobactam in ventilator-associated pneumonia (VAP). We present a case of VAP due to pan-resistant Pseudomonas aeruginosa in a patient with advanced multiple sclerosis. He was treated with ceftolozane/tazobactam in combination with colistin and rifampin for synergistic effect. Within two weeks of treatment, he had significant improvement in his leukocytosis and chest infiltrates, and his ventilator settings were adjusted to their baseline settings. This case illustrates the importance of using this novel antipseudomonal antibiotic to treat bacteria that are resistant to a wide spectrum of antibiotics, including carbapenems. Other antibiotics, like colistin and rifampin, can be used for synergism until more data are collected from trials evaluating the efficacy of monotherapy with this novel antibiotic for VAP.
\end{abstract}

Key words: pan-resistant Pseudomonas, novel antipseudomonal antibiotic, combination therapy

\section{INTRODUCTION}

Pseudomonas aeruginosa is responsible for a significant percentage of nosocomial infections, including pneumonia, bacteremia, and urinary tract infections. The ability of a single strain of $P$. aeruginosa

Corresponding author: Ammar Alqaid MD Contact Information: ammaralqaid@gmail.com DOI: $10.12746 /$ swrccc2015.0311.144 to acquire antimicrobial resistance via multiple mechanisms has made $P$. aeruginosa especially difficult to treat. ${ }^{1}$ Ceftolozane/tazobactam is a novel antibacterial agent and $\beta$-lactamase-inhibitor combination that has appreciable activity against wild-type Enterobacteriaceae and $P$. aeruginosa. Combination therapies based on polymyxin $E$ (colistin), rifampin, and carbapenems have been used in many cases to effectively treat multidrug resistant Gram negative infections. 


\section{CASE}

A 69-year-old man had progressive advanced multiple sclerosis. His respiratory system had been compromised by his disease, and he had undergone permanent tracheostomy. During the past year he became a resident of a nursing home and required assist-control mode ventilation with a backup rate of 12 , a tidal volume of $600 \mathrm{~mL}$, a positive end-expiratory pressure (PEEP) of $5 \mathrm{cmH}_{2} \mathrm{O}$, and a $\mathrm{FiO}_{2}$ of $40 \%$. $\mathrm{He}$ became tachypneic and somnolent and developed increased tracheostomy secretions. He was admitted to the medical intensive care unit; his initial work up revealed a WBC of $29.9 \mathrm{k} / \mu \mathrm{L}$ and urine WBC of 104/ HPF. His chest $\mathrm{x}$-ray showed bilateral interstitial infiltrates with a right lower lung field predominance (Figure 1). Empiric therapy with vancomycin and piperacillin/tazobactam was started. The patient required a backup rate of 20 and a PEEP of 10 to maintain oxygen saturation above 90 percent. On hospital day three the final urine culture grew Extended Spectrum Beta-Lactamase-producing (ESBL) Proteus mirabilis and Providencia stuartii (Figure 2). A culture of tracheostomy aspirate began growing Gram negative bacilli that were oxidase positive and non-lactose fermenting. Piperacillin/tazobactam was changed to meropenem, and vancomycin was stopped. By day five his WBC had dropped to $13 \mathrm{k} / \mathrm{\mu L}$, but he still required the same back up rate and PEEP on mechanical ventilation. His final tracheobronchial culture showed pan-resistant Pseudomonas aeruginosa that was also resistant to meropenem (Figure 3). The patient was started on ceftolozane/tazobactam 1000/500 mg IV every 8 hours, colistin $150 \mathrm{mg}$ IV every 12 hours, and rifampin $300 \mathrm{mg}$ every 12 hours via PEG tube. Within two weeks, his WBC decreased to $7 \mathrm{k} / \mu \mathrm{L}$, the back-up rate was decreased to 12, PEEP was decreased to 5 $\mathrm{cmH}_{2} \mathrm{O}$, and the chest $\mathrm{x}$-ray showed near complete resolution of infiltrates (Figure 4).

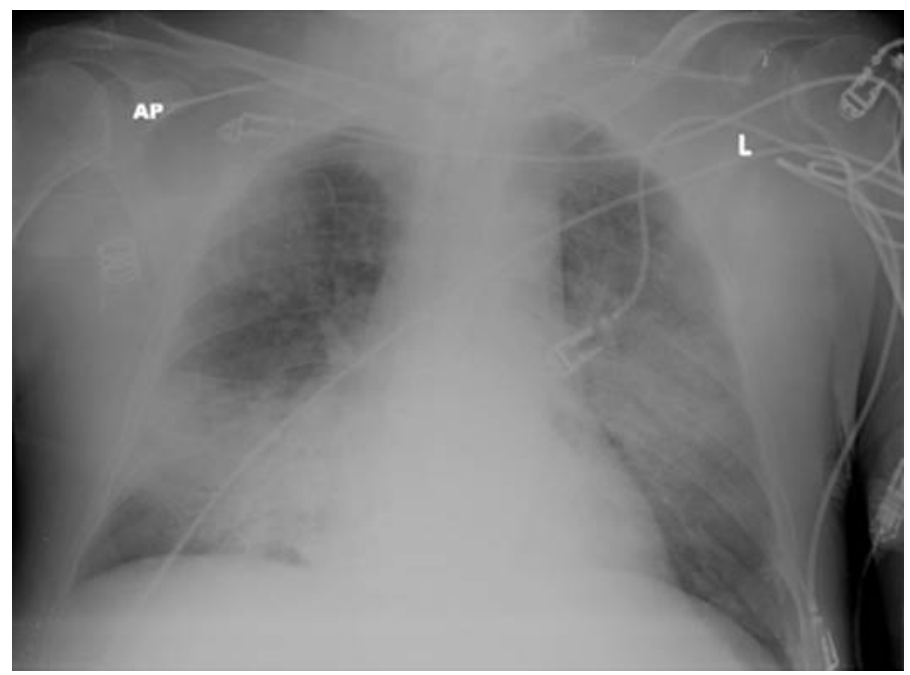

Figure1: Chest $\mathrm{x}$-ray on admission showing bilateral interstitial infiltrates, especially in the right lower lung field.

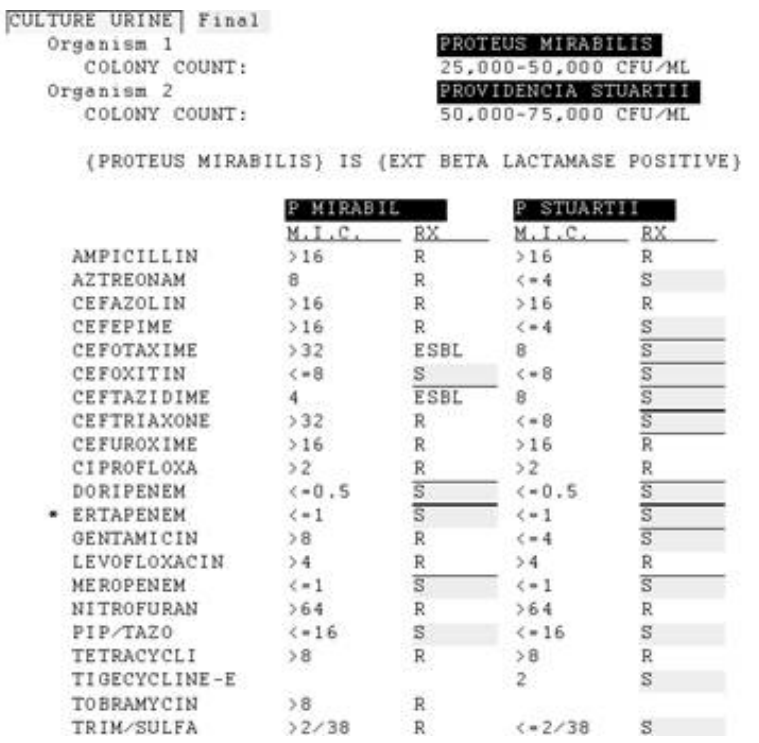

Figure2: Urine culture showing Proteus mirabilis (ESBL) and Providencia stuartii. 


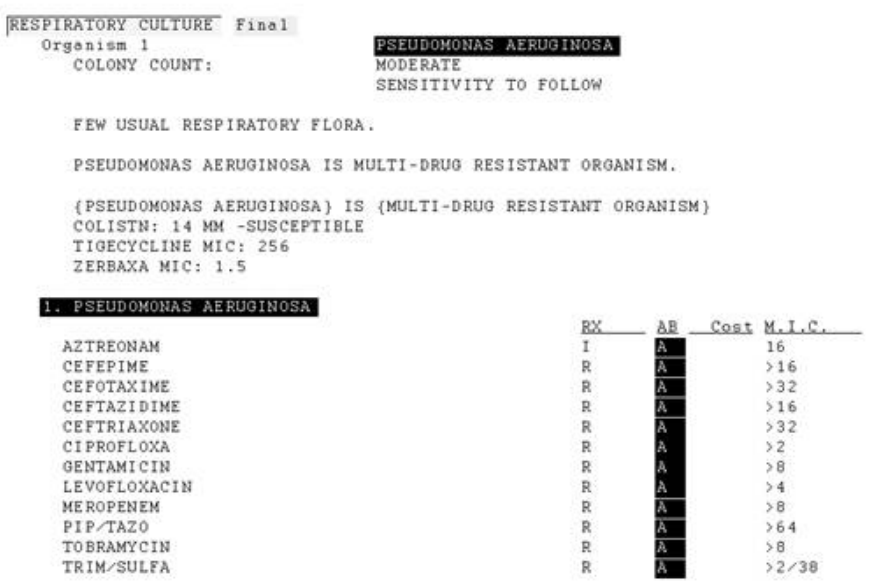

Figure3: Tracheobronchial aspirate culture showing pan-resistant Pseudomonas aeruginosa.

\section{Discussion}

Despite the wide distribution of $P$. aeruginosa in the environment, this microorganism rarely colonizes humans. However, the incidence of colonization increases significantly in hospitalized patients. In some hospitals, $P$. aeruginosa is the initial causative organism of infection, particularly in urinary and lower respiratory tract infections. Resistance of $P$. aeruginosa to antipseudomonal drugs has increased throughout the world. The mechanisms of resistance are complex and multifactorial and involve acquisition of genes (mainly against beta-lactams and aminoglycosides), chromosomal gene mutations (target site mutations affecting fluoroquinolones), and up-regulation of multidrug efflux pumps. Carbapenems have been one of the most important classes of antibiotics used in the empiric treatment of nosocomial infections, especially in severe cases. However, resistance of $P$. aeruginosa to imipenem and meropenem is increasing. The main mechanism of resistance to carbapenems in the United States is the loss of the OprD outer membrane porin protein. In other countries production of metallobeta-lactamases also has a major role. ${ }^{4,5}$

Ceftolozane/tazobactam (formerly CXA-201)

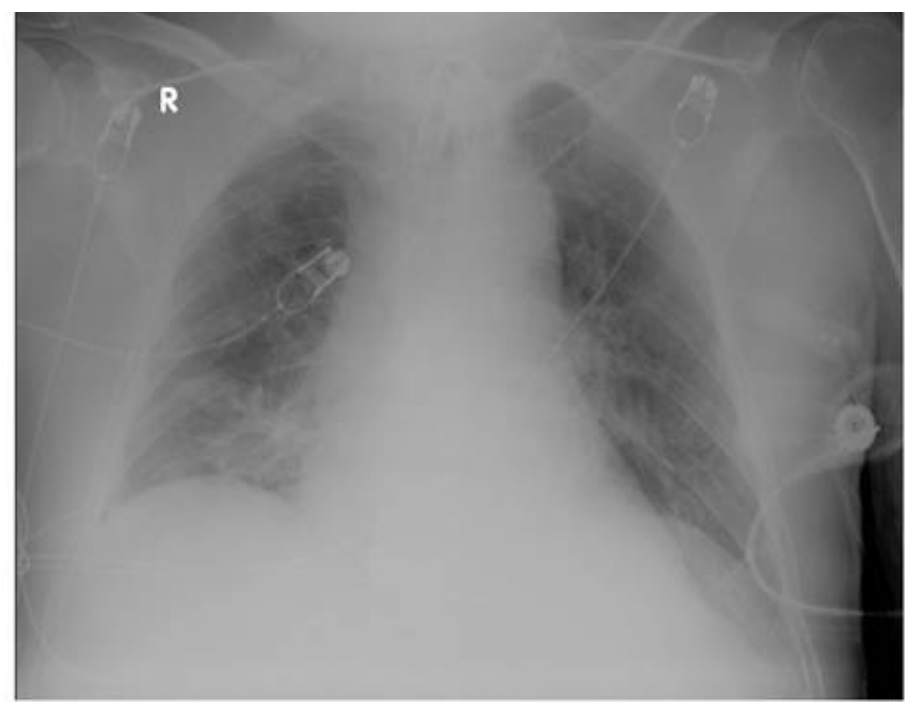

Figure4: Repeat chest x-ray after two weeks therapy with the triple antibiotics showed near complete resolution of infiltrates.

is a novel antibacterial and $\beta$-lactamase-inhibitor combination with the potential to meet the challenges created by multidrug resistant strains of $P$. aeruginosa. It has demonstrated good stability to AmpC $\beta$-lactamases and is less affected by changes in porin permeability and efflux pumps as a result of its strong binding of select penicillin-binding proteins (PBPs). ${ }^{6}$ More specifically, in comparison to ceftazidime and imipenem, ceftolozane has greater affinity for all essential PBPs (1b, 1c, 2, and 3). ${ }^{7}$

Currently, a trial is enrolling subjects in a prospective, randomized, double blind, multicenter, phase 3 clinical study to assess the safety and efficacy of IV ceftolozane/tazobactam compared to IV meropenem in adult patients with ventilated nosocomial pneumonia. Subjects receive either ceftolozane/tazobactam $3 \mathrm{~g}$ (administered IV over 60 minutes) every 8 hours for 8 days (14 days for Pseudomonas aeruginosa) or meropenem $1 \mathrm{~g}$ (administered IV over 60 minutes) every 8 hours for 8 days (14 days for P. aeruginosa). In part, the rationale for employing higher doses of ceftolozane stems from the results of an experimental rabbit pneumonia model that showed significantly 
greater reduction in pulmonary bacterial load with ceftolozane human-equivalent doses of $2000 \mathrm{mg}$ IV every 8 hours compared to ceftolozane human-equivalent doses of $1000 \mathrm{mg}$ IV every 8 hours. ${ }^{9}$ Ceftolozane/tazobactam $1.5 \mathrm{~g}$ administered every $8 \mathrm{~h}$ via a 60 minute infusion was used in our patient as acceptable plasma and epithelial lining fluid concentrations were achieved in a previous study with this dose. ${ }^{10}$ Our literature review found several studies citing the synergistic activity of dual combination therapy regimens consisting of colistin plus rifampin, colistin plus carbapenem, and rifampin plus carbapenem in the treatment of multidrug resistant Gram negative bacteria, but only one case report using triple combination therapy (colistin, rifampin and meropenem) has been described in the treatment of multidrug resistant $A$. baumannii infection. ${ }^{3}$

Our patient was already being treated with meropenem for his ESBL urinary tract infection, and his Pseudomonas isolate was resistant to the carbapenem class. The Pseudomonal sensitivity to colistin was evaluated by the disc susceptibility testing method, which showed a zone of inhibition of $14 \mathrm{~mm}$. Interpretative criteria for disc susceptibility testing of colistin are not available from the Clinical and Laboratory Standards Institute (CLSI) and interpretations of inhibition zone size are based on product literature. A previous study showed that disc diffusion remains an inherently unreliable susceptibility testing method and failed to detect colistin resistance compared to dilution-based methods, particularly for $P$. aeruginosa. We elected to use ceftolozane/tazobactam as the backbone of our antibiotic treatment for this case of pan-resistant Pseudomonas VAP since the isolate was sensitive to this antibiotic with a MIC of 1.5 (with a MIC cutoff limit of $4 \mathrm{mg} / \mathrm{L}$ considered to be resistant by the $E$ test strip dilution method) and that information was available within 5 days of sample collection. We added colistin and rifampin for synergistic effect. His creatinine was closely monitored, as nephrotoxicity has been reported in some cases with the use of intravenous colistin. Rifampin, when combined with colistin, has been shown to have in vitro and in vivo synergistic effects in the treatment of multidrug resistant Gram negative bacteria. ${ }^{12}$ In our patient, this triple combination therapy was effective and well tolerated.

\section{Conclusions}

Until controlled trials evaluating the effectiveness of monotherapy with novel antipseudomonal antibiotics like ceftolozane/tazobactam in the treatment of multidrug resistant Gram negative VAP are available, triple antibiotic therapy with the addition of colistin and rifampin for synergy is a promising option on a case by case basis.

\begin{abstract}
Author Affiliation: Ammar Alqaid and Christopher K. Dougherty are residents in Internal Medicine at Good Shepherd Medical Center, Longview/ University of Texas Health Science Center at Tyler, TX. Shahbaz Ahmad is an infectious disease physician at Good Shepherd Medical Center, Longview, TX.

Received: 05/09/2015

Accepted: 06/14/2015

Reviewers: Kristen Fuhrmann Pharm D

Published electronically: 07/15/2015

Conflict of Interest Disclosures: none
\end{abstract}

\section{REFERENCES}

1. Landman D, Bratu S, Alam M, Qualw J. Citywide emergence of Pseudomonas aeruginosa strains with reduced susceptibility to polymyxin B. J Antimicrob Chemother 2005 Jun; 55(6):954-7. 2. Hong M, Hsu D, Bounthavong M. Ceftolozane/tazobactam: a novel antipseudomonal cephalosporin and $\beta$-lactamase-inhibitor combination. Infect Drug Resist 2013 Nov 29; 6:215-23.

3. Biancofiorce G, Tasscini C, Bias M, et al. Colistin, meropenem and rifampin in a combination therapy for multi-drug-resistant Acinetobacter baumannii multifocal infection. Minerva Anestesiol 2007; 73:181-5. 
4. Castanheira M, Mills J, Farrell D, Jones R. Mutation-driven $\beta$-Lactam resistance mechanisms among contemporary ceftazidime-nonsusceptible Pseudomonas aeruginosa isolates from U.S. hospitals. Antimicrob Agents Chemother. 2014 Nov; 58(11): 6844-50.

5. Tuon F, Gortz L, Rocha J. Risk factors for pan-resistant Pseudomonas aeruginosa bacteremia and the adequacy of antibiotic therapy. Braz J Infect Dis 2012; 16(4):351-356.

6. Livermore D, Mushtaq S, Ge Y. Chequerboard titration of cephalosporin CXA-101 (FR264205) and tazobactam versus beta-lactamase-producing Enterobacteriaceae. J Antimicrob Chemother 2010; 65(9):1972-74.

7. Moyá B, Zamorano L, Juan C, Ge Y, Oliver A. Affinity of the new cephalosporin CXA-101 to penicillin-binding proteins of Pseudomonas aeruginosa. Antimicrob Agents Chemother 2010; 54(9):2933-37.

8. ClinicalTrials.gov. Safety and Efficacy of ceftolozane/tazobactam to treat ventilated nosocomial pneumonia (ASPECT-NP). Identifier NCT02070757. Accessed on July 29.2014.[weblink].

9. Jacqueline $\mathrm{C}$, Bretonniere $\mathrm{C}$, Desessard $\mathrm{C}$, et al. In vivo activity of CXA-101 against Pseudomonas aeruginosa (PA) in a rabbit experimental model of pneumonia: comparison with ceftazidime (CAZ), piperacillin/tazobactam (TZP), and imipenem (IMP). In: Abstracts: 51st Interscience Conference on Antimicrobial Agents and Chemotherapy. Washington: American Society for Microbiology; 2011.

10. Chandorkar G, Huntington J, Gotfried M, Rodvold K, Umeh $\mathrm{O}$.Intrapulmonary penetration of ceftolozane/tazobactam and piperacillin/tazobactam in healthy adult subjects. J Antimicrob Chemother 2012; 67:2463-69.

11. Tan T, Ng L. Comparison of three standardized disc susceptibility testing methods for colistin. Journal of Antimicrobial Chemotherapy (2006); 58: 864-867.

12. Tascini $C$, Ferranti $S$, Messina F, Menichetti F. In vitro and in vivo synergistic activity of colistin, rifampin and amikacin against a multidrug resistant $\mathrm{P}$. aeruginosa. Clin Microbiol Infect 2000; 6: 690-1. 